\title{
Prevalence of Nutrition and Exercise Counseling for Patients with Hypertension
}

\author{
United States, 1999 to 2000
}

Philip B. Mellen, MD, Shana L. Palla, MS, David C. Goff, Jr., MD, PhD, Denise E. Bonds, MD, MPH

OBJECTIVE: To evaluate the prevalence of nutrition and exercise counseling for patients with hypertension.

DESIGN: Cross-sectional survey data from the National Ambulatory Medical Care Survey (NAMCS) and the National Hospital Ambulatory Medical Care Survey (NHAMCS) for 1999 and 2000.

SETTING: Office-based physician practices and hospital outpatient departments.

PATIENTS/PARTICIPANTS: Patients age 18 or older with a diagnosis of hypertension.

MEASUREMENTS AND MAIN RESULTS: In 1999 and 2000, over 137 million patient encounters had a diagnosis of hypertension. Nutrition and exercise counseling were provided at $35 \%$ and $26 \%$ of visits, respectively. Patients older than 74 received the least nutrition (28\%) and exercise (18\%) counseling. Asians and Hispanics were more likely to undergo any lifestyle counseling, while non-Hispanic whites received the least exercise and nutrition counseling. Patients with 2 cardiovascular comorbidities were counseled for diet (53\%) and exercise (32\%) more than those with 1 (44\% and $31 \%$ ) or none (30\% and $23 \% ; P \leq .001$ and $P \leq .001)$. The encounters in the NAMCS had higher rates of counseling than those in the NHAMCS. There were no significant differences in counseling rates with respect to gender, geographic region, severity of hypertension, or physician specialty.

CONCLUSIONS: While there were significant differences in counseling rates with respect to age, race, comorbidity, and survey cohort, counseling for therapeutic lifestyle changes for patients with hypertension was uniformly suboptimal. Physicians need to recognize the importance of nonpharmacologic treatment in hypertension.

KEY WORDS: hypertension; lifestyle counseling; national survey.

J GEN INTERN MED 2004;19:917-924.

$\mathrm{C}$ ardiovascular disease, including stroke and heart disease, accounts for $37 \%$ of deaths in the United States. ${ }^{1}$ Hypertension is a significant risk factor for these diseases and represents a large health burden with a

Received from the Section of General Internal Medicine, Department of Internal Medicine (PBM, DCG, DEB), Section of Biostatistics, Department of Public Health Sciences (SLP), and Section of Epidemiology, Department of Public Health Sciences (DCG, DEB), Wake Forest University School of Medicine, WinstonSalem, NC.

Address correspondence and requests for reprints to Dr. Bonds: Section of Epidemiology, Department of Public Health Sciences, Wake Forest University, School of Medicine, Medical Center Boulevard, Winston-Salem, NC 27157 (e-mail: dbonds@wfubmc.edu). prevalence of $23 \%$ in the United States. ${ }^{2}$ The World Health Organization (WHO) estimates suboptimal blood pressure to be the number one attributable risk for death worldwide. ${ }^{3}$ Treatment of hypertension lowers cardiovascular morbidity and mortality, but studies have shown that only $29 \%$ of patients with hypertension have adequately controlled blood pressure as recommended by the Joint National Committee on the Prevention, Detection, Evaluation, and Treatment of High Blood Pressure (JNC-VI). ${ }^{4}$ Numerous clinical trials have established that lifestyle interventions can lower blood pressure and circumvent or decrease the need for antihypertensive medications. ${ }^{5-17}$ Wellstudied lifestyle interventions include weight loss, ${ }^{5-10}$ sodium restriction, ${ }^{6,7,11,12}$ a diet rich in fruits and vegetables (DASH diet), ${ }^{11,13,14}$ limited alcohol intake, ${ }^{15,16}$ and regular aerobic exercise. ${ }^{17}$

The Joint National Committee's Seventh Report (JNC-7) recommends lifestyle modification, with or without pharmacologic treatment, for all patients with a blood pressure greater than $120 / 80 .{ }^{18}$ Other national and international health organizations, including the American Heart Association and the WHO, recommend that physicians help patients at risk for cardiovascular disease set dietary and physical activity goals to reduce cardiovascular risk. ${ }^{19,20}$ Several researchers have found a low prevalence of counseling for diet and exercise by physicians both for primary prevention of heart disease ${ }^{21,22}$ and in groups with high-risk comorbidities such as diabetes, ${ }^{23,24}$ dyslipidemia, ${ }^{25}$ and obesity. ${ }^{26,27}$ While these studies included many patients with hypertension, none looked specifically at patients in the general population with high blood pressure. We sought to determine the prevalence of nutrition and exercise counseling for patients with hypertension and to determine whether specific patient, physician, or encounter characteristics alter that prevalence.

\section{METHODS}

\section{Data Source}

Data were compiled from the National Ambulatory Medical Care Survey (NAMCS) and the National Hospital Ambulatory Medical Care Survey (NHAMCS) for the years 1999 and 2000. These annual surveys are national probability samples conducted by the National Center for Health Statistics (NCHS) at the Centers for Disease Control and Prevention $(\mathrm{CDC})$. Using estimation procedures outlined by the NCHS, data from these surveys may be used to generate national population estimates. ${ }^{28}$ The NAMCS is a survey of U.S. office-based physicians who are selected 
randomly by specialty and geographic location from the master files of the American Medical Association and the American Osteopathic Association. Participating physicians record information on individual patient encounters during a randomly selected 1-week period. Physicians and office staff complete a single-page encounter form for each selected patient visit, which details patient demographic information, visit type, reasons for visit, diagnostic and screening services conducted or ordered during the encounter (special examinations, laboratory tests, or radiological studies), therapeutic and preventive services provided or ordered during the encounter (including counseling and other therapy), and new or continued medications prescribed or provided during the visit. Encounter forms also allow up to three International Classification of Diseases, Ninth Revision (ICD-9) diagnostic codes. Field staff perform checks for completeness and retrieve missing information from patient charts. Unobtainable demographic data (age, race, and gender) are imputed by the data processing service. Previous validation of the survey instrument has been published elsewhere. ${ }^{29}$

The NHAMCS samples visits to the outpatient departments and emergency rooms of nonfederally funded, noninstitutional, short-stay hospitals randomly selected from the SMG Hospital Market database based on geographic location. Selected hospitals participate on a rotating basis and visits are sampled over a 4 -week period. The survey instrument is identical to the NAMCS for parameters included in this study. Data from the outpatient department portion of the NHAMCS were used in this analysis.

\section{Dependent Variables}

We included all encounters with nonpregnant patients ages 18 years and older with an associated diagnosis of hypertension (ICD-9 codes 401.XX) and examined rates of nutrition and exercise counseling as marked in two separate check boxes under the Therapeutic and Preventive Services section of the instrument. Nutrition counseling was defined as the discussion of any topic related to the dietary intake of the patient to include general dietary guidelines, dietary instructions for specific medical conditions, or a referral to another health professional (e.g., dietician or nutritionist). Exercise counseling included any discussion of a patient's conditioning or fitness as well as referrals to other health or fitness professionals.

\section{Independent Variables}

Independent variables analyzed included patient and visit demographic information, including gender, age, race/ ethnicity, type of insurance (if any), geographic location, and visit type. Age was divided into groups: 18 to 44,45 to 64,65 to 74 , and $>74$. The survey instrument contains categories for both race (white, black, Asian, Pacific Islander, or Native American) and ethnicity (Hispanic or non-Hispanic). Encounters marked Hispanic were classified as Hispanic, regardless of race, and encounters marked as non-Hispanic were classified by the race marked. Due to extremely small sample sizes, Native Americans and Pacific Islanders were excluded. Insurance status (private insurance, Medicare, Medicaid, self-pay or uninsured, and other) was also examined. Because a previous NAMCS study found regional variations in care, ${ }^{22}$ geographic location of the encounter (Northeast, Midwest, South, or West) was examined. The primary reason for the visit (acute problem; chronic problem, routine; chronic problem, flare-up; or nonillness care) was also included.

The counseling rates in relation to cardiovascular comorbidities documented were also examined. Comorbidities examined included known coronary artery disease (ICD-9 codes 411.XX, 412.XX, 413.XX, 414.XX), diabetes mellitus (ICD-9 codes 250.XX), dyslipidemia (ICD-9 codes 272.XX), congestive heart failure (ICD-9 codes 402.XX, 428.XX), stroke (ICD-9 codes 434.XX, 436.XX), and obesity (ICD-9 codes 278.XX). Counseling rates for patients with 0,1 , or 2 of these comorbidities were compared. As the instrument allowed for only 3 diagnoses, including hypertension, those with more comorbidities would be listed as having 2 . The sample sizes for congestive heart failure $(\mathrm{CHF})$ and stroke were insufficient for individual analysis, but these were included in the cumulative comorbidity data.

The survey instruments include space for up to 6 current or new medications. These are subsequently given a 5-digit drug code corresponding to generic and brand name codes established by the NCHS. Medication codes falling into major antihypertensive classes (ACE-inhibitors/angiotensin II receptor blockers, alpha-blockers, beta-blockers, calciumchannel blockers, diuretics, centrally acting medications, and miscellaneous other antihypertensives) were identified and the number of antihypertensive medications for each encounter was determined. The number of antihypertensive medications $(0,1$ to 2 , or 3 or more) was used as a surrogate for the complexity of hypertension care.

The NAMCS identifies the specialty of the participating physician. To compare counseling patterns by specialty, rates were examined by the following specialties: internal medicine, family medicine, cardiology, and "other" specialties.

\section{Statistical Analysis}

Analyses were performed using STATA version 7.0 (STATA Corporation, College Station, Tex). Population averages for all demographic characteristics and treatments were determined using the survey commands in STATA. These commands account for the survey design and individual weighting of survey data. Preliminary analyses examined nutrition and/or exercise counseling differences in a variety of covariates. Survey logistic regression was performed to examine the relationship between counseling differences and provider type, geographic region, hypertension-related comorbidities, the number of hypertension-related comorbidities, the number of anti-hypertensive medications prescribed, and whether the encounter was from the NAMCS 
database or the NHAMCS database. The adjusted effect of these covariates was also examined using survey logistic regression. Specifically, we modeled the percentage of visits including nutrition or exercise counseling as a function of each covariate after adjusting for age group, gender, race/ ethnicity, payer source, and type of visit. $P$ values reflect the significance of the covariate's estimated coefficient in the adjusted model. We also calculated odds ratios for patients with specific cardiovascular comorbidities compared to patients without a given comorbidity.

\section{RESULTS}

In the 1999 and 2000 surveys, 6,696 patient encounters included a diagnosis of hypertension, representing approximately 137 million visits (Table 1). Non-Hispanic whites accounted for the majority of visits (69\%), followed by African Americans (17\%), Hispanics (8\%), and Asians (6\%). Women accounted for 58\% of the sample estimate. More visits were made by those ages 45 to 64 years (39\%) than by those ages 65 to 74 (26\%), over 74 (25\%), or 18 to $44(11 \%)$. The majority of patients represented had private insurance $(42 \%)$ or Medicare $(40 \%)$.

Overall counseling rates were $35 \%$ for diet (Table 2) and $26 \%$ for exercise (Table 3). There was no difference in counseling between men (diet 36\%, exercise 26\%) and women (diet $35 \%$, exercise $25 \%$ ). With respect to age, patients older than 74 received the least amount of diet (28\%) and exercise (18\%) counseling, with patients ages 18 to 44 receiving the most $(39 \%, P=.025$ and $30 \%, P=.003$, respectively). There were significant differences with respect to race: Asians (diet 50\%, exercise 44\%) and Hispanics (diet 52\%, exercise $47 \%$ ) were counseled most, while nonHispanic whites (diet 31\%, exercise 22\%) were counseled least (diet $P \leq .001$, exercise $P \leq .002$ ). Patients with Medicaid had the highest exercise counseling rates (34\%), while patients with Medicare and "other" payment providers had lower rates $(22 \%$ and $21 \% ; P=.05)$. Dietary counseling rates with respect to payment source revealed no significant difference. Acute visits were less likely to include nutrition or exercise counseling than chronic routine or chronic flare $(P \leq .001$ for diet and .005 for exercise for visittype comparisons). There were no significant differences in counseling rates by geographic region.

Counseling rates differed with respect to different cardiovascular risk factors or comorbidities. Patients with a diagnosis of diabetes (odds ratio [OR], 1.65; 95\% confidence interval [CI], 1.31 to 2.08), obesity (OR, 4.29; 95\% CI, 2.98 to 6.17 ), or dyslipidemia (OR, 1.50; 1.02 to 2.22 ) were more likely to receive dietary counseling than patients without these diseases. Obesity was the only identified cardiovascular comorbidity with significantly higher odds of receiving exercise counseling (OR, 3.91; 95\% CI, 2.53 to 6.02). Patients with 2 cardiovascular comorbidities were counseled for diet (53\%) and exercise (32\%) more than those with 1 (diet 44\%, exercise $31 \%$ ) or none (diet $30 \%$, exercise $23 \%$; adjusted $P \leq .001$ and $\leq .001$ for all numbers of comorbidity comparisons, respectively). The number of antihypertensive medications, a surrogate for severity of hypertension, was not associated with increased rates of counseling.

The two surveys showed disparate counseling rates as well. The NAMCS, reflecting office-based practices, had diet and exercise counseling rates of $36 \%$ and $27 \%$, respectively. These were significantly greater than rates seen in the NHAMCS, at hospital-based clinics, where 25\% and $14 \%$ of visits included counseling for diet and exercise $(P \leq .001)$.

Of the 124 million visits estimated by the NAMCS, there were no significant differences in counseling rates with respect to physician specialty. There was a trend toward more dietary counseling from family physicians (41\%) than from internists $(35 \%)$ or cardiologists $(23 \% ; P=.07)$. The trend was similar for exercise counseling (family medicine $30 \%$, internal medicine $28 \%$, cardiology $17 \%$, other $18 \% ; P=.09$ ).

\section{DISCUSSION}

Despite national guideline recommendations and compelling evidence for the efficacy of lifestyle changes for primary or adjunctive treatment of hypertension, we found low percentages of visits associated with a diagnosis of hypertension that included counseling for nutrition (35\%) or exercise (26\%). This is consistent with earlier NAMCS studies that showed a similarly low prevalence of lifestyle counseling for patients with modifiable cardiovascular risk factors. ${ }^{24,26}$ There were significant differences in counseling rates with respect to race, age, payment source, and type of visit as well as individual and cumulative cardiovascular comorbidities.

African Americans bear a greater burden from hypertension than do their non-Hispanic white counterparts, ${ }^{30}$ and Hispanic patients with hypertension have worse blood pressure control rates than non-Hispanic whites. ${ }^{31}$ We found higher rates of counseling for all minorities, though African Americans received counseling for exercise at rates similar to non-Hispanic whites. This could reflect physician awareness of racial disparities in hypertension control and perceived baseline lifestyle differences.

Elderly patients $(>74)$ were least likely to receive diet or exercise counseling, despite evidence for the benefit of lifestyle interventions for older hypertensive patients. ${ }^{10}$ This difference was only seen in the oldest age group and could be secondary to a higher percentage of debilitated patients in this population, but could also reflect practitioners who fail to recognize the benefit of diet and activity modulation in older patients. ${ }^{32}$ Type of visit was also associated with counseling rates: chronic routine visits had the highest counseling rates while acute care visits had the lowest. This is not surprising as counseling related to hypertension might be best addressed in routine follow-up for chronic illness. Insurance status had less association with counseling rates, with significant differences found only for exercise counseling. Of note, encounters with Medicaid or private insurance payment sources had higher 
Table 1. Characteristics of Visits with a Diagnosis of Hypertension (1999 to 2000 Estimates)

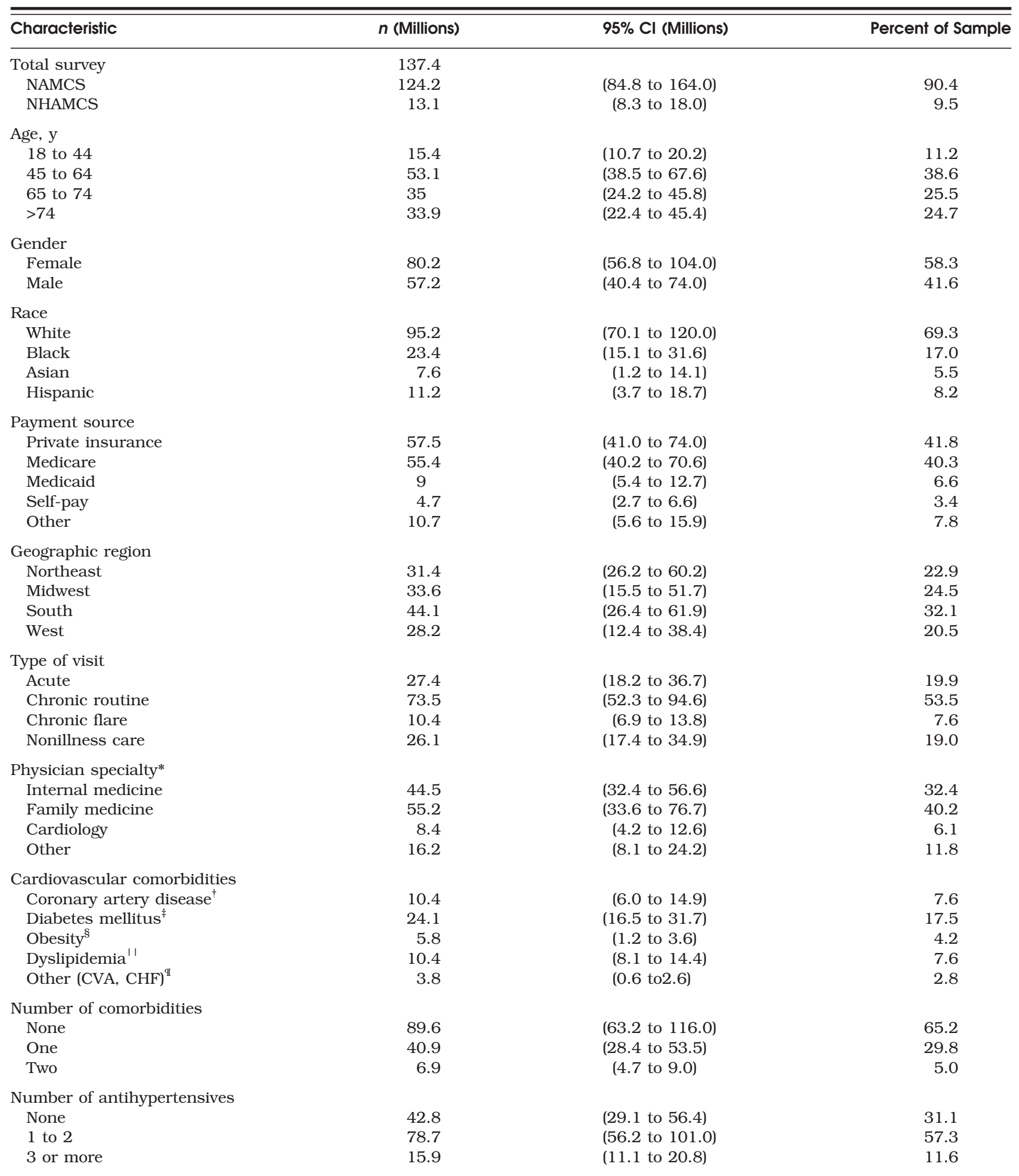

\footnotetext{
* Data for NAMCS only.
}

${ }^{\dagger}$ ICD 9 codes: 411.1, 411.81, 411.89, 412, 413.0, 413.1, 413.9, 414.00 to 414.05 .

${ }^{\ddagger}$ ICD 9 codes: 401.0, 401.1, 401.9.

§ ICD 9 code: 278.00 .

"ICD 9 codes: 272.1 to 272.9 .

IIICD 9 codes: 434.0, 434.1, 436, and 428.0, 428.1, 428.9 402.01, 402.11, 402.91.

NAMCS, National Ambulatory Medical Care Survey; NAHMCS, National Hospital Ambulatory Medical Care Survey; CVA, stroke; CHF, congestive heart failure; CI, confidence interval. 
Table 2. Estimated Diet Counseling Rates in Outpatient Visits with a Diagnosis of Hypertension

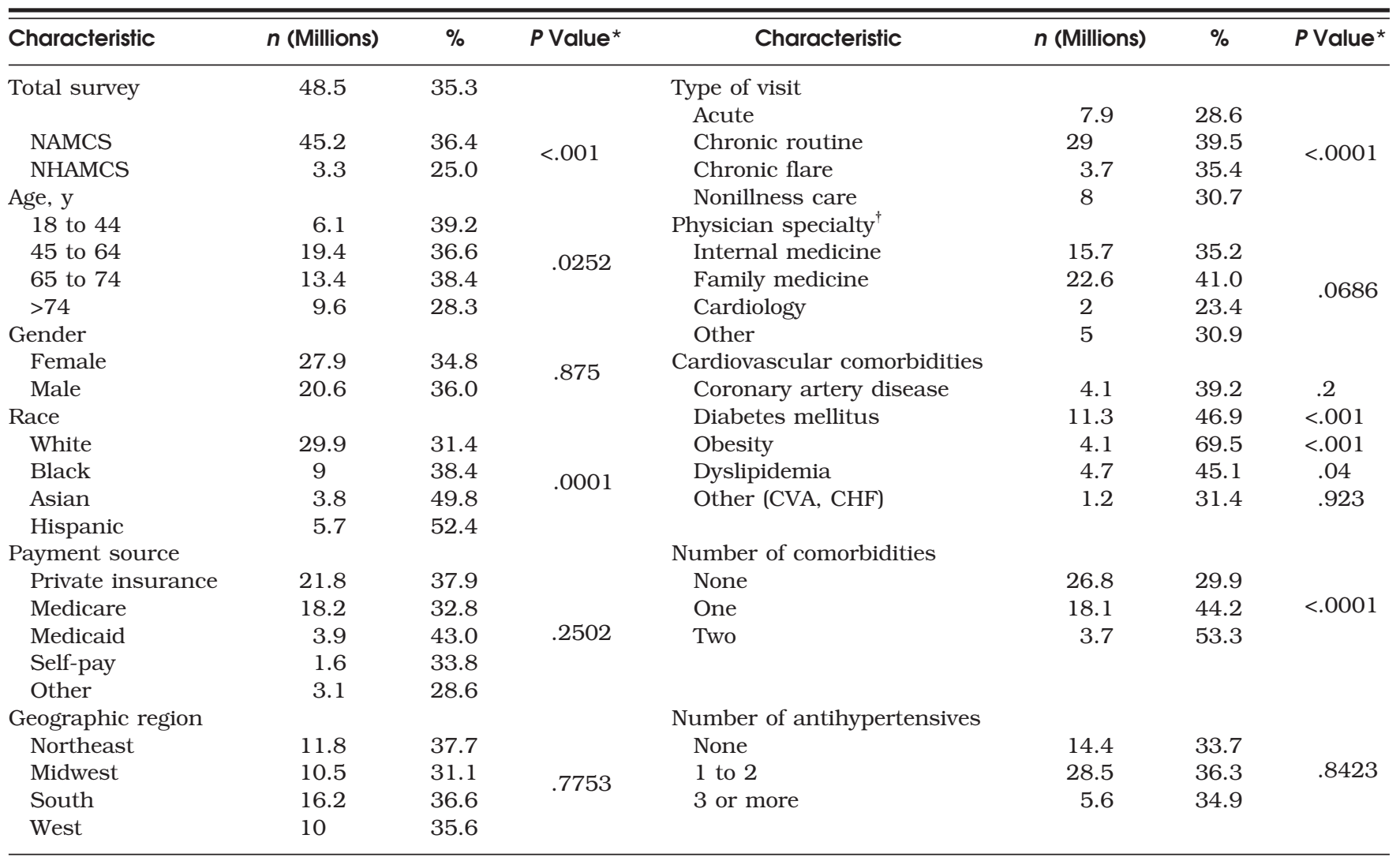

* Adjusted for age, gender, race/ethnicity, payment source, and type of visit.

${ }^{\dagger}$ Data from NAMCS only.

NAMCS, National Ambulatory Medical Care Survey; NAHMCS, National Hospital Ambulatory Medical Care Survey; CVA, stroke; CHF, congestive heart failure.

counseling rates while Medicare visits less often involved exercise counseling. This points once more to lower counseling rates for older patients.

While visits that identified diabetes, hyperlipidemia, or obesity all involved more nutrition counseling, obesity was the only comorbidity associated with higher exercise counseling rates. This difference is likely exaggerated, however, by the probable underreporting of obesity in the sample: while the concurrent National Health and Nutrition Examination Survey (NHANES) 1999 to 2000 found an age-adjusted prevalence of obesity to be $31 \%,{ }^{33}$ obesity was identified in only $4 \%$ of our projected population. This underestimation could be attributed to the number of comorbidities associated with obesity and the limit of 3 diagnoses associated with each visit. Given that our population had hypertension, it is likely that the prevalence of obesity in this group would be higher than the general population.

We found a striking disparity between the encounters from the NAMCS and the NHAMCS. The sample design of the NHAMCS is weighted toward a more urban population, and the NHAMCS has a higher percentage of self-pay and lower percentage of privately insured patients than the NAMCS. ${ }^{34,35}$ However, the significant difference in coun- seling rates persisted when adjusted for age, race, gender, payment source, and type of visit. This may reflect a difference in time and counseling resources in an office-based versus a hospital-based practice. An additional factor may be the presence of medical residents' participation in the NHAMCS and not in the NAMCS. Previous studies have shown that residents in training are more attuned to medication management and feel less confident approaching lifestyle counseling. ${ }^{36}$ Furthermore, the nature of residency training frequently truncates the longitudinal relationship in which effective lifestyle intervention can take place. A training physician may finally establish a therapeutic alliance and set intervention goals shortly before the resident completes her training and care of the patient is passed on to another physician.

The JNC-7 recommends a diet rich in fruits, vegetables, and low-fat dairy products alongside decreased intake of saturated fat, total fat, and dietary sodium $(<6 \mathrm{~g}$ of sodium chloride). This should be complemented by regular aerobic physical activity for at least 30 minutes a day most days of the week, with a concurrent goal of maintaining a normal body mass index $(<25) .{ }^{18}$ These guidelines are consistent with other national and international recommendations for patients with hypertension or otherwise increased 
Table 3. Estimated Exercise Counseling Rates in Outpatient Visits with a Diagnosis of Hypertension

\begin{tabular}{|c|c|c|c|c|c|c|c|}
\hline Characteristic & $n$ (Millions) & $\%$ & $P$ Value* & Characteristic & $n$ (Millions) & $\%$ & $P$ Value* \\
\hline Total & 35.3 & 25.7 & & Type of visit & & & \multirow{4}{*}{.0455} \\
\hline survey & & & & Acute & 6 & 21.9 & \\
\hline NHAMCS & 18.2 & 13.8 & $<.001$ & Chronic flare & 2.6 & 25.5 & \\
\hline Age, $y$ & & & & Nonillness care & 6.5 & 24.9 & \\
\hline 18 to 44 & 4.7 & 30.4 & \multirow{2}{*}{.0027} & Physician specialty $^{\dagger}$ & & & \multirow{2}{*}{.0876} \\
\hline$>74$ & 6.2 & 18.4 & & Cardiology & 1.5 & 17.3 & \\
\hline Gender & & & \multirow{3}{*}{.655} & Other & 2.9 & 18.1 & \\
\hline Female & 20.2 & 25.2 & & Cardiovascular comorbidities & & & \\
\hline Male & 15.1 & 26.4 & & Coronary artery disease & 3 & 28.4 & .331 \\
\hline Race & & & \multirow{2}{*}{.0024} & Diabetes mellitus & 6.7 & 28.0 & .675 \\
\hline White & 21.4 & 22.4 & & Obesity & 3.4 & 56.9 & $<.001$ \\
\hline Private insurance & 16.8 & 29.2 & \multirow{5}{*}{.0493} & None & 20.3 & 22.7 & \multirow{5}{*}{.0002} \\
\hline Medicare & 12.1 & 21.8 & & One & 12.7 & 31.2 & \\
\hline Medicaid & 3.1 & 34.1 & & Two & 2.3 & 33.0 & \\
\hline Self-pay & 1.1 & 23.1 & & & & & \\
\hline Other & 2.3 & 21.0 & & & & & \\
\hline Geographic region & & & \multirow{5}{*}{.6066} & Number of antihypertensives & & & \multirow{5}{*}{.6355} \\
\hline Northeast & 7.3 & 23.2 & & None & 10.4 & 24.4 & \\
\hline Midwest & 8.1 & 24.1 & & 1 to 2 & 20.7 & 26.3 & \\
\hline South & 11.7 & 26.5 & & 3 or more & 4.2 & 26.6 & \\
\hline West & 8.2 & 29.1 & & & & & \\
\hline
\end{tabular}

* Adjusted for age, gender, race/ethnicity, payment source, and type of visit.

${ }^{\dagger}$ Data from NAMCS only

NAMCS, National Ambulatory Medical Care Survey; NAHMCS, National Hospital Ambulatory Medical Care Survey; CVA, stroke; CHF, congestive heart failure.

cardiovascular risk. ${ }^{19,20,37}$ However, despite agreement on dietary and activity recommendations, the best way to implement these counseling interventions is unclear. Previous physician surveys have found that, although primary care providers recognize the importance of healthy lifestyle choices, they are generally pessimistic about patients' ability to change behavior. ${ }^{38-41}$ Other significant barriers to counseling include lack of time for or training in lifestyle counseling. ${ }^{38}$ The intensive interventional models in most clinical trials involve multiple follow-up visits. For dietary interventions, the U.S. Preventive Services Task Force (USPSTF) found that more intensive interventions and those of longer duration yielded greater and more sustained benefit. ${ }^{42}$ Replicating the intensive follow-up found in most large nutrition and fitness interventional trials would be problematic when applied to the large population with hypertension, given the current explosion of health care costs and diminishing reimbursement.

An alternative approach could focus on more individualized patient-based counseling interventions. The transtheoretical (or "stages of change") model recognizes individuals in different states in relationship to behavioral changes-precontemplation, contemplation, preparation, action, maintenance, and termination. ${ }^{43}$ Using this frame- work, clinicians can identify readiness for change and tailor counseling to encourage progress toward action and maintenance. ${ }^{44}$ Thus, with limited targeted intervention, patients may be more likely to progress to action. ${ }^{45}$ Another technique, used more frequently in addiction medicine, is motivational interviewing, in which the clinician elicits from the patient the reasons for changing behavior. ${ }^{46,47}$ This method yields results similar to much more intensive and confrontational methods of behavioral counseling. Both of these approaches illustrate how a patient-centered intervention that emphasizes the individual decision to change may enable significant change without intensive interventions. While these more sophisticated behavioral techniques may not infiltrate the breadth of primary care in the immediate future, the inclusion of these concepts in residency patient communication skills training may help cultivate a generation of physicians more confident and competent in behavioral counseling. This could compliment more widespread use of group counseling led by nonphysicians (nutritionists, fitness instructors) or physician extenders (nurses, nurse practitioners, physician assistants).

This study has several strengths, including the large sample size and national representation of the data. It is limited by the cross-sectional design of the survey. The 
measured unit in these surveys was a single patient visit. Patients with hypertension often have multiple visits for management, presenting multiple opportunities for counseling. Thus, our sample may underestimate the incidence of lifestyle counseling over a period of time. If the average patient with hypertension had quarterly office visits as outlined by JNC-7 guidelines, ${ }^{18}$ a random cross-section of visits should find a prevalence of diet and exercise counseling of $25 \%$ in patients counseled annually. Indeed, Desai et al. found lifestyle counseling rates of greater than $80 \%$ over a 2-year period in a Veteran's Administration population with hypertension and/or obesity. ${ }^{27}$ However, lifestyle changes once effected are best maintained within the context of a longitudinal relationship, and some degree of counseling should take place within each physician-patient encounter. Another limitation of our study lies in the reliance of the surveys on chart extraction by field staff for missing information. Previous studies have shown medical records to be less sensitive measures of health habit counseling, ${ }^{48}$ and thus our findings may underestimate the number of visits in which counseling was included.

The survey instrument does not include specific information on the content of lifestyle intervention counseling, and thus a casual encouragement to decrease sodium intake would constitute an intervention, though the efficacy would surely be different from a more formal nutritional intervention. ${ }^{42}$ Furthermore, the limit of 3 diagnoses limits the sensitivity of our study in looking at patients with hypertension and other comorbidities, that is, patients with multiple diagnoses and hypertension may not have been included in the study, and patients with hypertension may not have been identified as having other cardiovascular risk factors if they had noncardiovascular diagnoses. Given the increase in counseling rates with greater comorbidity, we would anticipate that inclusion of these patient encounters might increase the observed prevalence of counseling. The 6-medication limit on the survey instrument may lead to the omission of some antihypertensive therapies, which could result in a bias toward the null if patients on 3 or more antihypertensives were recognized as taking 2 or fewer.

We looked solely at patients with a diagnosis of hypertension, and thus counseling efforts constituted secondary prevention. The increasing prevalence of obesity ${ }^{33}$ as well as an increase in the intake of salty, calorie-dense foods ${ }^{49}$ highlights the role for primary prevention of hypertension. The coordinating committee of the National High Blood Pressure Education Program (NHBPEP) recommends targeted lifestyle intervention for groups at high risk for the development of hypertension, including patients who are obese or overweight, have a family history of hypertension, are African American, consume excessive amounts of salt or alcohol, have inadequate potassium intake, or are sedentary. ${ }^{50}$ The Joint National Committee's Seventh Report (JNC-7) further highlights the need for identification of high-risk patients by the new classification of "prehypertension" for patients with systolic blood pressure of 120 to
139 or diastolic blood pressure of 80 to $89 .{ }^{18}$ In our study, patients with hypertension and no other identified cardiac risk markers received counseling much less frequently than patients with more advanced cardiac risk. Physicians should not only incorporate lifestyle counseling into their treatment for patients with hypertension, but also recognize other patients at risk and counsel accordingly.

\section{Conclusions}

Though lifestyle interventions are effective tools for lowering blood pressure, they remain underutilized in physician practice. While we found significant differences with respect to age, race, payment provider, visit type, cardiovascular comorbidity, and survey cohort, counseling rates were low throughout the sample. Physicians need to recognize the importance of the nonpharmacologic treatment of hypertension, and further work is needed to translate the knowledge gained from clinical trials into widespread clinical practice. This may include physician training in patient-centered counseling methods and increased funding for and utilization of adjunctive nutrition and fitness counselors.

\section{REFERENCES}

1. Anderson RN. Deaths: leading causes for 2000. Natl Vital Stat Rep. 2002;50:1-86.

2. Coresh J, Wei L, McQuillan G, et al. Prevalence of high blood pressure and elevated serum creatinine level in the United States: findings from the Third National Health and Nutrition Examination Survey (1988-1994). Arch Intern Med. 2001;161:1207-16.

3. World Health Report 2002: Reducing Risks, Promoting Healthy Life. Geneva Switzerland: World Health Organization; 2002. Available at: http//www.who.int/whr/2002. Accessed January 25, 2004.

4. The sixth report of the joint national committee on prevention, detection, evaluation, and treatment of high blood pressure. Arch Intern Med. 1997;157:2413-46.

5. Masuo K, Mikami H, Ogihara T, Tuck ML. Weight reduction and pharmacologic treatment in obese hypertensives. Am J Hypertens. 2001;14(6 pt 1):530-8.

6. He J, Whelton PK, Appel LJ, Charleston J, Klag MJ. Long-term effects of weight loss and dietary sodium reduction on incidence of hypertension. Hypertension. 2000;35:544-9.

7. Effects of weight loss and sodium reduction intervention on blood pressure and hypertension incidence in overweight people with high-normal blood pressure: the Trials of Hypertension Prevention, Phase II. The Trials of Hypertension Prevention Collaborative Research Group. Arch Intern Med. 1997;157:657-67.

8. Stevens VJ, Obarzanek E, Cook NR, et al. Trials for the Hypertension Prevention Research Group. Long-term weight loss and changes in blood pressure: results of the Trials of Hypertension Prevention, Phase II. Ann Intern Med. 2001;134:1-11.

9. Imai Y, Sato K, Abe K, et al. Effect of weight loss on blood pressure and drug consumption in normal weight patients. Hypertension. 1986;8:223-8.

10. Whelton PK, Appel LJ, Espeland MA, et al. Sodium reduction and weight loss in the treatment of hypertension in older persons: a randomized controlled trial of nonpharmacologic interventions in the elderly (TONE). TONE Collaborative Research Group. JAMA. 1998;279:839-46.

11. Sacks FM, Svetkey LP, Vollmer WM, et al. DASH-Sodium Collaborative Research Group. Effects on blood pressure of reduced dietary 
sodium and the Dietary Approaches to Stop Hypertension (DASH) diet. N Engl J Med. 2001;344:3-10.

12. Appel LJ, Espeland MA, Easter L, Wilson AC, Folmar S, Lacy CR. Effects of reduced sodium intake on hypertension control in older individuals: results from the Trial of Nonpharmacologic Interventions in the Elderly (TONE). Arch Intern Med. 2001;161:685-93.

13. Appel LJ, Moore TJ, Obarzanek E, et al. A clinical trial of the effects of dietary patterns on blood pressure. N Engl J Med. 1997;336: 1117-24.

14. Appel L, Champagne CM, Harsha DW, et al. Writing Group of the PREMIER Collaborative Research Group. Effects of comprehensive lifestyle modification on blood pressure: results of the PREMIER clinical trial. JAMA. 2003;289:2083-93.

15. Xin X, He J, Frontini MG, Ogden LG, Motsamai OI, Whelton PK. Effects of alcohol reduction on blood pressure: a meta-analysis of randomized controlled trials. Hypertension. 2001;38:1112-7.

16. Puddey IB, Parker M, Beilin LJ, Vandongen R, Masarei JRL. Effects of alcohol and caloric restrictions on blood pressure and serum lipids in overweight men. Hypertension. 1992;20:533-41.

17. Whelton SP, Chin A, Xin X, He J. Effect of aerobic exercise on blood pressure: a meta-analysis of randomized, controlled trials. Ann Intern Med. 2002;136:493-503.

18. Chobanian AV, Bakris GL, Black HR, et al. National Heart, Lung, and Blood Institute Joint National Committee on Prevention, Detection, Evaluation, and Treatment of High Blood Pressure; National High Blood Pressure Education Program Coordinating Committee. Seventh report of the Joint National Committee on Prevention, Detection, Evaluation, and Treatment of High Blood Pressure. Hypertension. 2003;42:1206-52.

19. Pearson TA, Blair SN, Daniels SR, et al. AHA Guidelines for Primary Prevention of Cardiovascular Disease and Stroke: 2002 update: consensus panel guide to comprehensive risk reduction for adult patients without coronary or other atherosclerotic vascular diseases. American Heart Association Science Advisory and Coordinating Committee. Circulation. 2002;106:388-91.

20. World Health Organization, International Society of Hypertension Writing Group. 2003 World Health Organization (WHO)/International Society of Hypertension (ISH) statement on management of hypertension. J Hypertens. 2003;21:1983-92.

21. Wee CC, McCarthy EP, Davis RB, Phillips RS. Physician counseling about exercise. JAMA. 1999;282:1583-8.

22. Giles W. Missed opportunities in preventive counseling for cardiovascular disease: United States, 1995. MMWR 1998;47:91-5.

23. Egede LE, Zheng D. Modifiable cardiovascular risk factors in adults with diabetes: prevalence and missed opportunities for physician counseling. Arch Intern Med. 2002;162:427-33.

24. Meigs JB, Stafford RS. Cardiovascular disease prevention practices by U.S. physicians for patients with diabetes. J Gen Intern Med. 2000;15:220-8.

25. Stafford RS, Blumenthal D, Pasternak RC. Variations in cholesterol management practices of U.S. physicians. J Am Coll Cardiol. 1997;29:139-46.

26. Stafford RS, Farhat JH, Misra B, Schoenfeld DA. National patterns of physician activities related to obesity management. Arch Fam Med. 2000;9:631-8.

27. Desai MM, Rosenheck RA, Druss BG, Perlin JB. Receipt of nutrition and exercise counseling among medical outpatients with psychiatric and substance use disorders. J Gen Intern Med. 2002;17:556-60.

28. National Center for Health Statistics. Ambulatory Health Care Data: Survey Methodology. Available at: http://www.cdc.gov/nchs/ about/major/ahcd/ahcd1.htm. Accessed September 30, 2003.

29. Burt CW. Use of geographic contextual variables in examining survey item validity. Seventh Conference on Health Survey Research
Methods. September 24-27, 1999. Available at: http://www.cdc.gov/ nchs/data/conf/conf07.pdf. Accessed January 10, 2004.

30. Cooper R, Rotimi C. Hypertension in blacks. Am J Hypertens. 1997;10(7 pt 1):804-12.

31. Crespo CJ, Loria CM, Burt VL. Hypertension and other cardiovascular disease risk factors among Mexican Americans, Cuban Americans, and Puerto Ricans from the Hispanic Health and Nutrition Examination Survey. Public Health Rep. 1996;111(suppl 2):7-10.

32. Pahor M, Applegate WB. Recent advances. Geriatric medicine. BMJ. 1997;315:1071-4.

33. Flegal KM, Carroll MD, Ogden CL, Johnson CL. Prevalence and trends in obesity among US adults, 1999-2000. JAMA. 2002;288: 1723-7.

34. Ly N, McCraig L, Burt CW. National Hospital Ambulatory Medical Care Survey: 1999 outpatient department summary. Adv Data Vital Health Stat. 2001;321:1-28.

35. Cherry DK, Woodwell DA. National Medical Care Survey: 2000 summary. Adv Data Vital Health Stat. 2002;328:1-32.

36. Rogers LQ, Bailey JE, Gutin B, et al. Teaching resident physicians to provide exercise counseling: a needs assessment. Acad Med. 2002;77:841-4

37. Expert Panel on Detection, Evaluation, and Treatment of High Blood Cholesterol in Adults. Executive Summary of the Third Report of the National Cholesterol Education Program (NCEP) Expert Panel on Detection, Evaluation, and Treatment of High Blood Cholesterol in Adults (Adult Treatment Panel III). JAMA. 2001;285:2486-97.

38. Kushner RF. Barriers to providing nutrition counseling by physicians: a survey of primary care practitioners. Prev Med. 1995;24:546-52.

39. Orleans CT, George LK, Houpt JL, Brodie KH. Health promotion in primary care: a survey of U.S. family practitioners. Prev Med. 1985; 14:636-47.

40. Yeager KK, Donehoo RS, Macera CA, Croft JB, Heath GW, Lane MJ. Health promotion practice among physicians. Am J Prev Med. 1996; 12:238-41.

41. Sherman SE, Hershman WY. Exercise counseling: how do general internists do? J Gen Intern Med. 1993;8:243-8.

42. U.S. Preventive Services Task Force. Behavioral counseling in primary care to promote a healthy diet. Am J Prev Med. 2003;24:93100.

43. Prochaska JO, Velicer WF. The transtheoretical model of health behavior change. Am J Health Promot. 1997;12:38-48.

44. Mann J. Importance of dietary management and practical patient counseling, the European/Australasian perspective. Atheroscler Suppl. 2002;3:23-9.

45. Calfas KJ, Long BJ, Sallis JF, Wooten WJ, Pratt M, Patrick K. A controlled trial of physician counseling to promote the adoption of physical activity. Prev Med. 1996;25:225-33.

46. Miller WR. Motivational interviewing: research, practice, and puzzles. Addict Behav. 1996;21:835-42.

47. Emmons KM, Rollnick S. Motivational interviewing in health care settings. Opportunities and limitations. Am J Prev Med. 2001;20:68-74.

48. Stange KC, Zyzanski SJ, Smith TF, et al. How valid are medical records and patient questionnaires for physician profiling and health services research? A comparison with direct observation of patient visits. Med Care. 1998;36:851-67.

49. Nielsen SJ, Popkin BM. Patterns and trends in food portion sizes, 1977-1998. JAMA. 2003;289:450-3.

50. Whelton PK, He J, Appel LJ, et al. National High Blood Pressure Education Program Coordinating Committee. Primary prevention of hypertension: clinical and public health advisory from the National High Blood Pressure Education Program. JAMA. 2002;288:1882-8. 